\title{
Degradación hidrolítica de recubrimientos polisiloxánicos de fibras de vidrio
}

\author{
M. JIMÉNEZ; J. GONZÁLEZ-BENITO; A.J. AZNAR; J. BASELGA \\ Dpto. Ciencia de Materiales, Univ. Carlos III de Madrid, Leganés (SPAIN)
}

\begin{abstract}
Se silanizaron fibras de vidrio mediante la utilización de disoluciones acuosas de tres silanos: 3-aminopropiltrietoxisilano (APES), 3-aminopropilmetildietoxisilano (APDES) y 3-aminopropildimetiletoxisilano (APMES). Se injertó químicamente una molécula fluorescente (cloruro de pirenosulfonilo, PSC) mediante la formación de una sulfonamida por reacción del grupo sulfonilo del PSC con los grupos amino de los recubrimientos. Se estudió el fenómeno de degradación hidrolítica del recubrimiento silano a $\mathrm{pH}=7$ en función de: i) la temperatura y ii) el tipo de recubrimiento. Se ha propuesto un mecanismo para la reacción de hidrólisis del polisiloxano enlazado a las fibras de vidrio y se ha obtenido la entalpía del proceso, cuyo valor fue de $3.5 \mathrm{kcal} / \mathrm{mol}$. También se ha observado que a medida que la funcionalidad del silano aumenta, la degradación hidrolítica es más rápida.
\end{abstract}

Palabras clave: Fibras de vidrio, aminosilanos, región de acoplamiento, marcadores fluorescentes, degradación hidrolítica.

Hydrolytic Degradation of Polysiloxanic Coatings on Glass Fibers

Aqueous solutions of three silanes: 3-aminopropyltriethoxysilane (APES), 3-aminopropylmethyldiethoxysilane (APDES), and 3-aminopropyldimethylethoxysilane (APMES), were used to cover glass fibers. The pyrenesulfonyl chloride fluorescent moiety (PSC) was chemically attached to the silanized substrate via the sulfonamide formation when the sulfonyl group of the PSC reacts with the amino residues of the coatings. It was studied the silane layer hydrolytic degradation phenomenon $(\mathrm{pH}=7)$ was studied as a function of: i) temperature, and ii) coating layer type. A hydrolysis reaction mechanism of the polysiloxane bonded onto the glass fibers has been proposed and, the enthalpy change of the process was obtained (3.5 kcal/ mol). In addition, it was observed that the greater the silane functionality the faster the hydrolytic degradation.

Key words: Glass fibers, aminosilanes, coupling region, fluorescent labels, hydrolytic degradation.

\section{INTRODUCCIÓN}

Uno de los componentes de refuerzo más utilizados para fabricar materiales compuestos de matriz polimérica son las fibras de vidrio. Debido a su baja resistencia a la abrasión, se tratan superficialmente con formulaciones químicas complejas, entre las que se pueden incluir como principales constituyentes, lubricantes y promotores de adhesión o agentes de acoplamiento. Cuando las fibras de vidrio están recubiertas de esta manera, se obtiene una mejora en las propiedades del material compuesto, especialmente en la adhesión, durabilidad y propiedades físicas de la interfase fibra/matriz polimérica (1-4).

Entre la gran variedad de agentes de acoplamiento existentes, los alcoxisilanos y en particular los que poseen grupos amino, tienen mucha importancia. Los agentes de acoplamiento silano se utilizan comúnmente para enlazar polímeros a vidrios, sílices u otros sustratos de alta superficie específica, siendo el campo de los materiales compuestos uno de los más importantes, ya que se consigue contrarrestar, entre otros, los efectos adversos del agua en sus propiedades mecánicas $(3,4)$

La mayor parte de la investigación referente a plásticos reforzados con fibras de vidrio se ha centrado en el estudio de cinco categorías:

i) Superficie de la fibra de vidrio ii) Interfase fibra de vidrio/agente de acoplamiento

iii) Agente de acoplamiento

iv) Interfase agente de acoplamiento/matriz polimérica

v) Matriz polimérica

Se ha mostrado en muchos casos, normalmente cuando la matriz polimérica no es muy hidrofílica y su resistencia a la hidrólisis es grande, que la calidad y durabilidad de material compuesto de matriz orgánica depende fundamentalmente de la naturaleza del anclaje y de la estructura en la región de acoplamiento, así como de la estabilidad de la interfase en entornos húmedos (3-5), es decir de la estabilidad de la región compuesta por las zonas ii), iii) y iv) citadas anteriormente.

Hasta el momento, la mayor parte de la investigación realizada en este campo se ha centrado en el estudio de la naturaleza del anclaje y la estructura del agente de acoplamiento sobre el material reforzante, utilizándose para ello multitud de técnicas instrumentales (6) tales como: RMN, SIMS, XPS, FTIR $(7,8)$, SEM (8), Fluorescencia $(7,9)$, medidas de ángulos de contacto dinámico (8), etc. También se han realizado medidas de propiedades mecánicas comparando el material seco con el mojado con agua. En este último apartado, generalmente, los estudios realizados a nivel molecular no han tenido mucho éxito $(10,11)$, de ahí que sea necesario obtener más y mejor información. 
La presencia de agua interfacial en materiales compuestos con fibras de vidrio como elementos reforzantes tiene un profundo efecto en la durabilidad de estos materiales. La optimización de las formulaciones de agentes de acoplamiento ha de tener en cuenta no sólo aspectos estructurales sino también la resistencia a la hidrólisis de los recubrimientos de las fibras, estando ambos aspectos íntimamente relacionados.

Recientemente se ha encontrado interesante combinar silanos di y trifuncionales para modificar el grado de entrecruzamiento con objeto de modificar la rigidez de la región de acoplamiento (12). Se ha encontrado que la presencia de silanos difuncionales mejora la resistencia interfacial aumentando la transmisión de tensiones interfaciales. Poco se sabe, sin embargo, acerca de la modificación de la difusividad del agua en la región de acoplamiento al modificar su estructura y de la modificación de sus efectos hidrolíticos.

En este trabajo se pretende medir la velocidad de hidrólisis, a pH controlado, de recubrimientos poliorganosiloxánicos en fibras de vidrio tratadas con silanos mono, di y trifuncionales. Para ello se emplea un nuevo método que permite medir con extremada sensibilidad todo el proceso de degradación en el que la concentración de silano en la disolución de hidrólisis es menor de $10^{-9} \mathrm{M}$. Este método se basa en la medida de la respuesta fluorescente de un cromóforo (pirenosulfonamida). Inicialmente esta especie está anclada al polisiloxano que recubre a las fibras de vidrio. Cuando el polímero se hidroliza los fragmentos creados pasan a la disolución junto con el cromóforo cuya fluorescencia es lo suficientemente intensa como para proporcionar información precisa de su concentración.

\section{PARTE EXPERIMENTAL}

\subsection{Materiales}

Las fibras de vidrio E fueron suministradas por VETROTEX. Se emplearon tres aminosilanos suministrados por ABCR como agentes de acoplamiento para recubrir las fibras de vidrio: 3-aminopropiltrietoxisilano (APES), 3-aminopropilmetildietoxisilano (APDES) y 3-aminopropildimetiletoxisilano (APMES), sin purificación previa. El marcador fluorescente utilizado fue cloruro de pireno-1-sulfonilo (PSC) suministrado por Molecular Probes. Los disolventes empleados, suministrados por Aldrich, fueron de calidad HPLC o superior. En todos los casos se emplearon tal y como fueron recibidos sin posteriores purificaciones excepto en el caso del tolueno que fue destilado sobre sodio para eliminar agua. Todos los experimentos de degradación hidrolítica se llevaron a cabo en una disolución acuosa a pH controlado (disolución tampón, potasio di-hidrógeno fosfato/di-sodio hidrógeno fosfato de $\mathrm{pH}=7$, Panreac Química S.A).

\subsection{Preparación de muestras}

\subsubsection{PRETRATAMIENTO DE FIBRAS}

Las fibras de vidrio comerciales, antes de ser silanizadas, fueron calcinadas en un horno a $450^{\circ} \mathrm{C}$ durante $1 \mathrm{~h}$. Esta temperatura de calcinación se ha seleccionado de forma que, por una parte, sea lo suficientemente alta para que la oxidación de la materia orgánica sea completa y, por otra, lo suficientemente baja como para evitar una deshidroxilación excesiva de la superficie (13). Después de la calcinación, las fibras de vidrio fueron sometidas a un tratamiento de activación mediante un reflujo de $\mathrm{HCl} 10 \%$ durante $3 \mathrm{~h}$ con el fin de regenerar los grupos silanol superficiales.

\subsubsection{SILANIZACIÓN}

Se introdujo 1 gramo de fibras de vidrio activadas en $50 \mathrm{ml}$ de disolución acuosa al 1\% (v/v) de: i) APMES (muestra A); ii) APDES (muestra B) y iii) APES (muestra C) a temperatura ambiente durante $10 \mathrm{~min}$. Después del correspondiente proceso de silanización, las fibras de vidrio fueron sometidas a un proceso de calentamiento a $110^{\circ} \mathrm{C}$ durante una hora para conseguir la policondensación de los residuos etoxi además de un secado. Posteriormente, dichas fibras secas fueron sometidas a un proceso de extracción en soxhlet con tolueno seco con el fin de eliminar todo el silano que no hubiera reaccionado. Por último, se procedió a un secado a vacío a temperatura ambiente durante al menos $8 \mathrm{~h}$.

\subsubsection{PIRENACIÓN}

Las fibras de vidrio silanizadas fueron marcadas con cloruro de pirenosulfonilo (PSC). Para ello, el cromóforo fue anclado a las fibras vía la formación de una sulfonamida (PSA) con las funcionalidades amina procedentes del recubrimiento poliorganosiloxánico. El tratamiento consistió en: a) sumergir $0.4 \mathrm{~g}$ de fibras silanizadas en $25 \mathrm{ml}$ de una disolución de marcador $\left(10^{-4} \mathrm{M}\right)$ en acetonitrilo durante $10 \mathrm{~min}$. a temperatura ambiente, b) un proceso de extracción en soxhlet con tolueno seco para eliminar cromóforo absorbido y c) un secado a vacío durante al menos $8 \mathrm{~h}$ a temperatura ambiente.

\subsection{Técnicas instrumentales}

Los espectros de fluorescencia fueron realizados en un fluorímetro LS-B50 de Perkin Elmer a una velocidad de 120 nm/ min y se utilizaron anchuras de rendija de 5 y $7 \mathrm{~nm}$ para excitación y emisión, respectivamente. Los experimentos de hidrólisis se realizaron introduciendo las fibras de vidrio en una cubeta para fluorimetría con la disolución tampón correspondiente. La temperatura se controló con un criostato, haciendo circular agua a temperatura constante a través de una camisa de refrigeración en el compartimento de muestra. La disolución se mantuvo en todo momento con agitación. Posteriormente, y de manera sucesiva, se realizaron espectros de emisión de la disolución en función del tiempo. Los espectros de las fibras marcadas se realizaron mediante excitación frontal.

Con objeto de relacionar la intensidad de fluorescencia de la especie PSA con su concentración, se realizó una curva de calibrado utilizando la intensidad de fluorescencia del PSA relativa a la de una muestra patrón (ovaleno).

Como $\mathrm{I}_{\mathrm{A}}=2.3 \cdot \mathrm{I}_{0} \varepsilon \mathrm{l}[\mathrm{A}]$, donde $\mathrm{I}_{\mathrm{A}}$ es la intensidad de fluorescencia de la especie A a una longitud de onda determinada $(\lambda \varepsilon), \mathrm{I}_{0}$ la intensidad de la luz con que se excita la muestra, $\varepsilon$ el coeficiente de extinción molar de la especie A a $\lambda \varepsilon, 1$ la longitud de paso óptico y [A] la concentración de la especie $\mathrm{A}$. Al dividir $\mathrm{I}_{\mathrm{PSA}}$ por la intensidad del patrón, tendríamos que:

$$
\frac{I_{P S A}}{I_{\text {ovaleno }}}=b[P S A]
$$

donde $b$ es una constante que sólo depende de la temperatura. Los valores de b obtenidos de esta manera se presentan a continuación:

\begin{tabular}{lllll}
\hline $\mathrm{T}(\mathrm{K})$ & 283 & 293 & 303 & 313 \\
$\mathbf{b x 1 0}^{-7}(\mathbf{l} / \mathbf{m o l})$ & 2.52 & 2.42 & 2.34 & 2.22
\end{tabular}




\section{RESULTADOS Y DISCUSIÓN}

\subsection{Caracterización de la región de acoplamiento}

\subsubsection{ESPECTROS DE EMISIÓN}

En la figura 1 se encuentran representados los espectros de emisión de todas las muestras preparadas (A, B y C). En todos los casos se observa fluorescencia. La asignación de las bandas para este tipo de muestras aparece detallada en otro trabajo publicado con anterioridad (9). Se puede observar que, a medida que la funcionalidad del silano empleado para realizar el recubrimiento aumenta, la banda ancha situada a unos $470 \mathrm{~nm}$ también lo hace. Esta banda fue asignada a la emisión de exciplejo (9). La formación de este exciplejo proviene de la interacción existente entre los grupos amino del recubrimiento y el grupo PSA excitado. Este resultado sugiere una mayor concentración de aminas a nivel local y, por tanto, un mayor grado de recubrimiento, al pasar de recubrimientos con monoetoxi a recubrimientos con di- y tri-etoxisilano.

\subsubsection{CROMATOGRAFÍA EN CAPA FINA}

La realización de cromatografía en capa fina del producto de hidrólisis, de una disolución acuosa de APES y de una disolución de PSC en acetonitrilo reveló dos manchas fluorescentes, presentando mayor retención la correspondiente al producto de hidrólisis. Dicha mancha se sitúa a la altura de la correspondiente a la disolución de APES. Este resultado sugiere que el producto de hidrólisis procede de la rotura de enlaces siloxano y no del grupo sulfonamida.

\subsection{Mecanismo de hidrólisis}

En la figura 2 se puede observar el conjunto de espectros de emisión correspondiente a un experimento de hidrólisis representativo. Se puede observar como, a medida que avanza el tiempo de reacción, la intensidad de fluorescencia aumenta, indicativo de la incorporación de la especie PSA a la disolución.

En la figura 3 se representa para la muestra C, a tres temperaturas, la variación de la relación de intensidades, IPSA/ Iovaleno, normalizada, en función del tiempo de permanencia de las fibras en la disolución. Se puede observar que, en los primeros instantes, la incorporación de PSA a la disolución es mucho más rápida, mientras que a tiempos altos de reacción, la intensidad de fluorescencia (la concentración) tiende a ser constante. Por otro lado, al comparar los experimentos a distintas temperaturas se observa que la velocidad de hidrólisis aumenta al aumentar la temperatura. Se observa la misma tendencia si se analizan los resultados en términos de concentraciones.

Para analizar el proceso de hidrólisis es necesario plantear las siguientes hipótesis:

i) La hidrólisis no es un proceso controlado por difusión. El coeficiente de difusión del agua medido en una atmósfera de $9 \% \mathrm{HR}$, es del orden de $\mathrm{D}=10^{-12} \mathrm{~m}^{2} \mathrm{~s}^{-1}$ a temperatura ambiente (10). Si se supone un espesor, L, de la capa de organosilano del orden de $10 \mathrm{~nm}$, el tiempo característico de difusión, $\tau$, se puede estimar en $10^{-4} \mathrm{~s}$. Parece evidente que la capa poliorganosiloxánica no debe comportarse como una barrera física contra la hidrólisis.

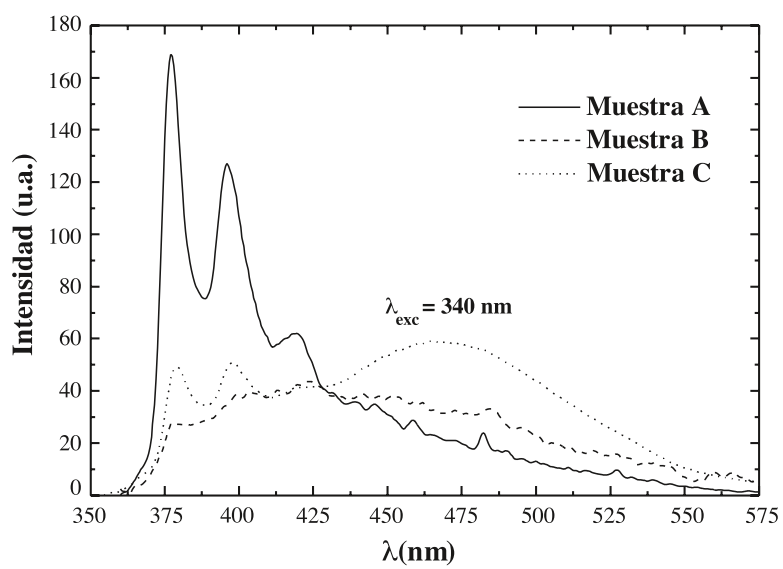

Figura 1.- Espectros de emisión fluorescente de fibras de vidrio silanizadas marcadas con PSA.

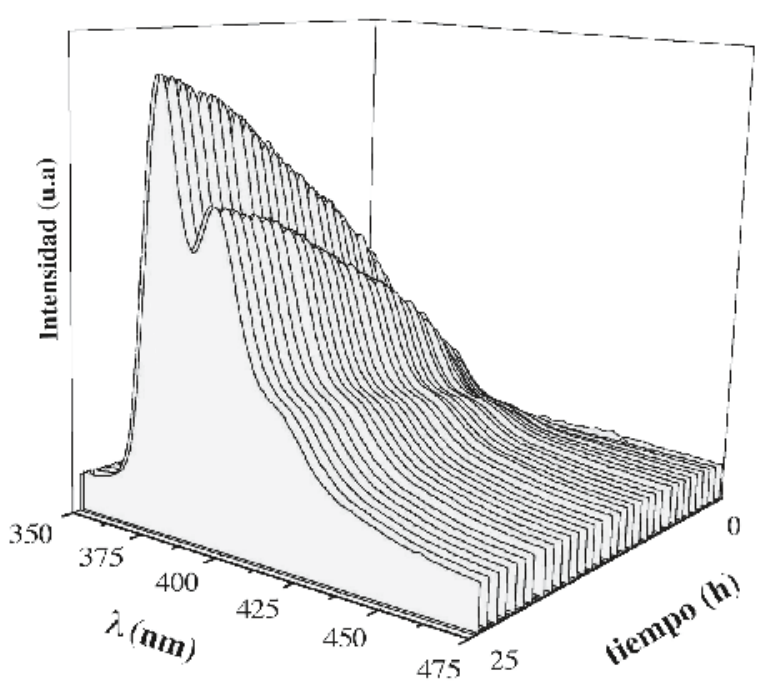

Figura 2.- Espectros de emisión correspondientes a un experimento de hidrólisis representativo.

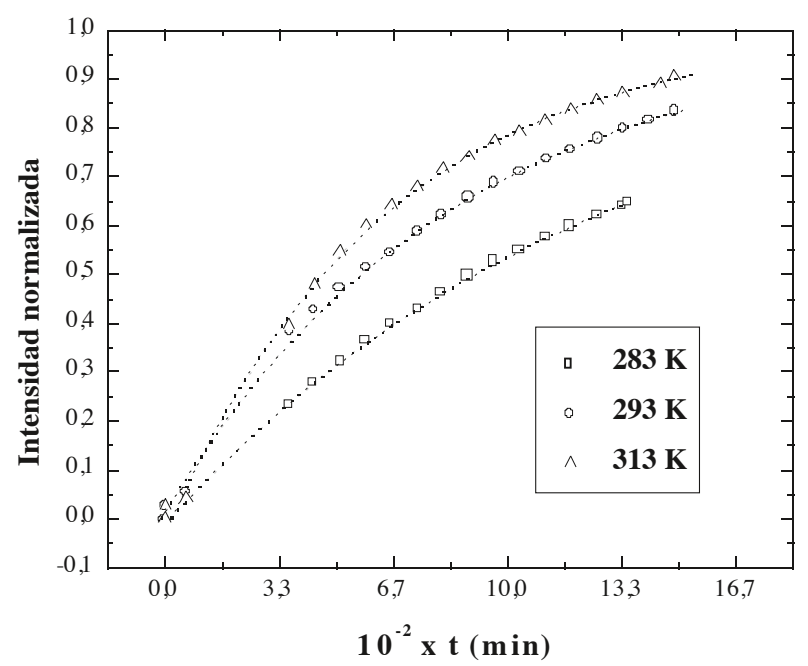

Figura 3.- Variación de la relación de intensidades $\mathrm{I}_{\mathrm{PSA}} / \mathrm{I}_{\mathrm{ovaleno}^{\prime}}$ normalizada en función del tiempo de permanencia de las fibras en la disolución. Muestra silanizada con APES (muestra C). 
ii) La hidrólisis de siloxanos es una reacción de equilibrio

$$
\mathrm{Si}-\mathrm{O}-\mathrm{Si} \leftrightarrow(\mathrm{Si}-\mathrm{OH})_{\mathrm{f}}+(\mathrm{Si}-\mathrm{OH})_{1}
$$

donde $\mathrm{Si}-\mathrm{O}-\mathrm{Si}$ es un enlace siloxano, $(\mathrm{Si}-\mathrm{OH})_{\mathrm{f}}$ es un grupo silanol producto de hidrólisis que permanece fijo en la estructura polisiloxánica sobre las fibras de vidrio y $(\mathrm{Si}-\mathrm{OH})$ un grupo silanol, producto de hidrólisis, que queda libre en disolución.

iii) la concentración de PSA es directamente proporcional a la concentración de grupos $(\mathrm{Si}-\mathrm{OH})_{1}$.

iv) Las velocidades de reacción en disolución dependen del $\mathrm{pH}$. Como aquí se considera una hidrólisis homogénea en un sólido, este parámetro es inherente a la estructura química de la red y no depende de la actividad protónica de la disolución que lo rodea, al menos antes de la conversión crítica de hidrólisis donde la red "colapsa" (10). Hay que tener en cuenta, que aunque la constante de velocidad del proceso directo, $\mathrm{k}_{\mathrm{d}^{\prime}} \mathrm{y}$ del proceso inverso, $\mathrm{k}_{\mathrm{i}}$, vienen afectadas por catálisis, se espera que no exista influencia en la relación $\mathrm{k}_{\mathrm{d}} / \mathrm{k}_{\mathrm{i}}$.

Según lo anterior la variación de la concentración de (Si$\mathrm{OH})_{1}$ en función del tiempo vendría dada por:

$$
v=\frac{d\left[(\mathrm{Si}-\mathrm{OH})_{l}\right]}{d t}=k_{d}[\mathrm{SiOSi}]-k_{i}\left[(\mathrm{SiOH})_{f}\right]\left[(\mathrm{SiOH})_{l}\right]
$$

donde $[\mathrm{SiOSi}]=[\mathrm{SiOSi}]_{0}-\left[(\mathrm{SiOH})_{1}\right]$ con $[\mathrm{SiOSi}]_{0}$ concentración inicial de enlaces siloxano.

Para definir la concentración $[\mathrm{SiOH}]_{\mathrm{f}}$ es necesario tener en cuenta tres posibles mecanismos de incorporación de fragmentos (silano) a la disolución:

a) Fragmentos del tipo 1, siendo $\alpha$ la fracción de estos fragmentos:

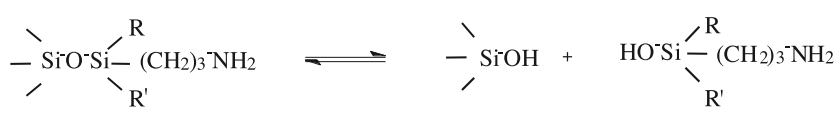

por tanto la contribución de este tipo de fragmentos a la concentración de $(\mathrm{SiOH})_{\mathrm{f}}$ será $\left([\mathrm{SiOH}]_{\mathrm{f} 0}+\alpha[\mathrm{SiOH}]_{\mathbb{l}}\right)$. Donde $[\mathrm{SiOH}]_{\mathrm{f} 0}$ es la concentración inicial de grupos silanol fijos.

b) Fragmentos del tipo 2, siendo $\beta$ la fracción de estos fragmentos:

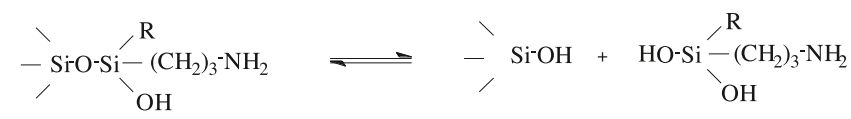

por tanto la contribución de este tipo de fragmentos a la concentración de $(\mathrm{SiOH})_{\mathrm{f}}$ será $\beta[\mathrm{SiOH}]_{\mathrm{f} 0}$

c) Fragmentos del tipo 3, siendo $\gamma$ la fracción de estos fragmentos:

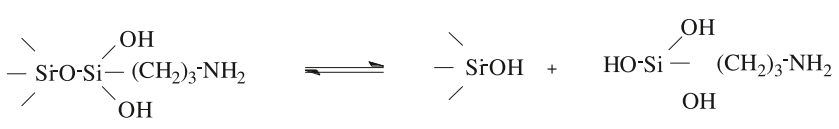

por tanto la contribución de este tipo de fragmentos a la concentración de $(\mathrm{SiOH})_{\mathrm{f}}$ será $\left([\mathrm{SiOH}]_{\mathrm{f} 0}-\gamma / 3[\mathrm{SiOH}]_{1}\right.$.

Según lo anterior, la ecuación (1) se convertirá en:

$$
\begin{aligned}
& v=\frac{d\left[(\mathrm{Si}-\mathrm{OH})_{l}\right]}{d t}=\quad \text { M. JIMÉNEZ; J. GONZÁLEZ-BENITO; A.J. AZNAR; I. BAS } \\
& \left.=k_{d}\left([\mathrm{SiOSi}]_{0}-[\mathrm{SiOH}]_{l}\right)-k_{i}\left([\mathrm{SiOH}]_{f 0}-\left(\frac{\gamma}{3}-\beta\right)[\mathrm{SiOH}]_{l}\right)[\mathrm{SiOH}]_{l}\right)= \\
& =k_{d}\left([\mathrm{SiOSi}]_{0}-[\mathrm{SiOH}]_{l}\right)+k_{i}\left(\frac{\gamma}{3}-\beta\right)[\mathrm{SiOH}]_{l}^{2}
\end{aligned}
$$

ecuación en la que el término $\left(\frac{\gamma}{3}-\beta\right)$ nos da idea del grado de entrecruzamiento del polímero que recubre a las fibras de vidrio y en la que se ha despreciado el término $\mathrm{k}_{\mathrm{i}}[\mathrm{SiOH}]_{\mathrm{f} 0}$ al suponerlo muy pequeño.

Al integrar la ecuación (2) y poner la concentración de $(\mathrm{SiOH})_{1}$ en función de la relación de intensidades y agrupar constantes en P1, P2, P3 y P4, obtenemos una expresión del tipo:

$$
t=P 1 \operatorname{Ln}\left[P 2 \frac{\left(P 3-\frac{I_{P S A}}{I_{\text {ovaleno }}}\right)}{\left(P 4-\frac{I_{P S A}}{I_{\text {ovaleno }}}\right)}\right]
$$

Utilizando la ecuación (3) se obtuvieron buenos ajustes de los datos experimentales de la figura 3, siendo P1, P2, P3 y P4 parámetros de ajuste. Dichos ajustes también aparecen en la figura 3 como líneas discontinuas. De los parámetros de ajuste se han podido obtener unas constantes aparentes de equilibrio, $\mathrm{K}_{\mathrm{eq}}\left(\frac{\gamma}{3}-\beta\right)$, para cada una de las temperaturas estudiadas.

\begin{tabular}{lc}
$\mathrm{T}(\mathrm{K})$ & $(\gamma / 3-\beta) \mathrm{K} \times 10^{5}$ \\
283 & 1.27 \\
\hline 293 & 1.63 \\
\hline 303 & 1.60 \\
\hline 313 & 2.50 \\
\hline
\end{tabular}

Teniendo en cuenta que:

$$
\ln \left(\frac{\gamma}{3}-\beta\right) K_{e q}=\left(\frac{\Delta S}{R}+\ln \left(\frac{\gamma}{3}-\beta\right)\right)-\frac{\Delta H}{R T}
$$

donde $\mathrm{T}$ es la temperatura en Kelvin, $\Delta S$ es la variación de entropía y $\Delta \mathrm{H}$ la de entalpía, al representar $\ln (\gamma / 3-\beta) \mathrm{K}_{\mathrm{eq}}$ vs $1 / \mathrm{T}$ se debería obtener una recta de cuya pendiente obtener $\Delta \mathrm{H}$.

En la figura 4 se representa la variación de $\ln (\gamma / 3-\beta) \mathrm{K}_{\text {eq }}$ en función de $1 / \mathrm{T}$ junto con un ajuste lineal con una correlación de 0.9 , lo cual es bastante aceptable. De los parámetros de

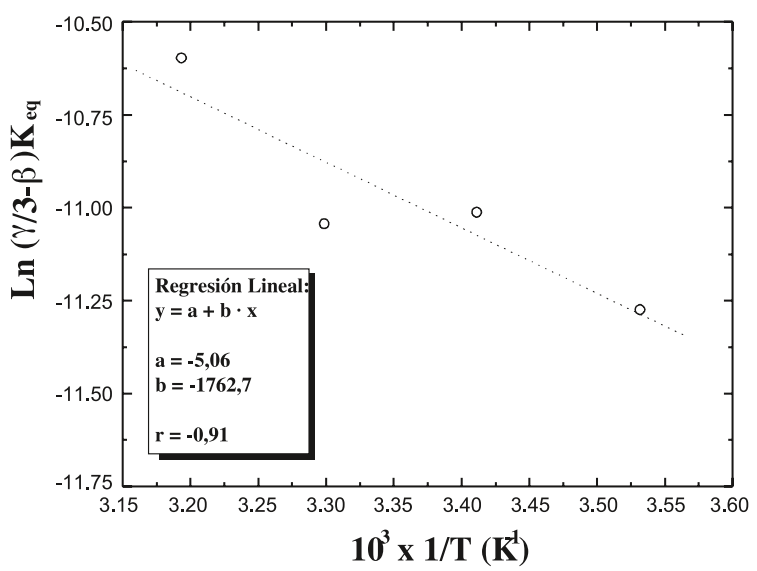

Figura 4.- variación de $\ln (\gamma / 3-\beta) \mathrm{K}_{\text {eq }}$ en función de $1 / \mathrm{T}$ junto con un ajuste lineal 
ajuste se ha podido obtener la entalpía de la reacción que resultó ser de $\Delta \mathrm{H}=3.5 \mathrm{kcal} \mathrm{mol}^{-1}$. Este resultado se encuentra dentro del mismo orden de magnitud correspondiente a la entalpía de solubilización de sílices (14).

\subsection{Hidrólisis en función del recubrimiento}

En la figura 5 se representa la variación de la relación de intensidades $\left(\mathrm{I}_{\mathrm{PSA}} / \mathrm{I}_{\text {ovaleno }}\right.$ ) normalizada, para las tres muestras en estudio, en función del tiempo de permanencia de las fibras en disolución. Se observa que el modelo cinético planteado para la degradación hidrolítica ajusta de manera aceptable los resultados experimentales, tal y como reflejan las líneas discontinuas. Por otro lado, a medida que la funcionalidad del silano aumenta de mono- a trietoxi-, la degradación hidrolítica de la región de acoplamiento es más rápida. Estos resultados sugieren que la rotura de enlaces siloxánicos entre la superficie de la fibra de vidrio y silano $\left(\mathrm{Si}_{\text {fibra }}-\mathrm{O}-\mathrm{Si}\right)$ por efecto del agua es más lenta que la rotura de enlaces siloxánicos entre silanos (Si-O-Si). El esquema de la figura 6 refleja con claridad la situación correspondiente a cada una de las muestras sometidas a estudio. Sólo las muestras B y C poseen enlaces del tipo Si-O-Si, de ahí que en estos casos la velocidad de incorporación de fragmentos silano a la disolución sea mayor. Además, el hecho de que las curvas correspondientes a esta muestras sean tan próximas sugiere que la muestra $C$ debe poseer un bajo grado de reticulación.

Teniendo en cuenta lo anterior, se podría concluir que una manera de reducir la velocidad de degradación de la región de acoplamiento por efecto hidrolítico consistiría en minimizar el grado de recubrimiento, es decir, reducir el número de enlaces Si-O-Si.

\section{CONCLUSIÓN}

El proceso de degradación hidrolítica de la región de acoplamiento en fibras de vidrio silanizadas ocurre por rotura de enlaces siloxánicos. Se ha propuesto un mecanismo de hidrólisis que da lugar a una ecuación integrada de velocidad que ajusta de manera aceptable los resultados experimentales. Según dicho mecanismo, la hidrólisis del siloxano es una reacción de equilibrio. Se ha medido la entalpía del proceso de hidrólisis para el APES sobre fibras de vidrio, obteniéndose un valor de $3.5 \mathrm{kcal} / \mathrm{mol}$. A medida que la funcionalidad del silano aumenta de mono- a trietoxi-, la degradación hidrolítica de la región de acoplamiento es más rápida.

\section{BIBLIOGRAFÍA}

1. K.L. Mittal, Silanes and Other Coupling agents, JSP, Ed. Utrech, (1992).

2. W. Noll, Chemistry and Technology of Silicones, Academic Press, London, (1968).

3. P. Walker, Organosilanes as Adhesion Promoters, J. Adhesion Sci. Technol., 5, 4, 279-305, (1991).

4. P. Plueddemann, Silane Coupling Agents, 2nd ed., Plenum, New York, (1991).

5. J.Jancar, A.T. Dibenedetto, Fibre Reinforced Thermoplastic Composites for dentistry, J. Materials Sci.: Mat. Medicine, 4, 555-561, (1993).

6. N. Suzuki, H. Ishida, A Review on the Structure and Characterization Techniques of Silane/Matrix Interphases, Macromol. Symp., 108, 19-53, (1996).

7. J. González-Benito, J.C. Cabanelas, A.J. Aznar, M.R. Vigil, J. Bravo, J. Baselga, Surface Characterization of Silanized Glass Fibers by Labeling with Environmental Sensitive Fluorophores, J. Applied Polym. Sci., 62, 375-384, (1996).

8. J. González-Benito, A.J. Aznar y J. Baselga, Microstructural and wettability study of surface pretreated glass fibers, J. Materials Process. Tech., 00, 000, 1999.

9. J. González-Benito, J.C. Cabanelas, A.J. Aznar, M.R. Vigil, J. Bravo, J. Baselga, Pyrene-Doped Polyorganosiloxane Layers over Commercial Glass Fibers, J. Fluorescence, 9, 1, 51-57, (1999).

Boletín de la Sociedad Española de Cerámica y Vidrio. Vol. 39 Núm. 4 Julio-Agosto 2000

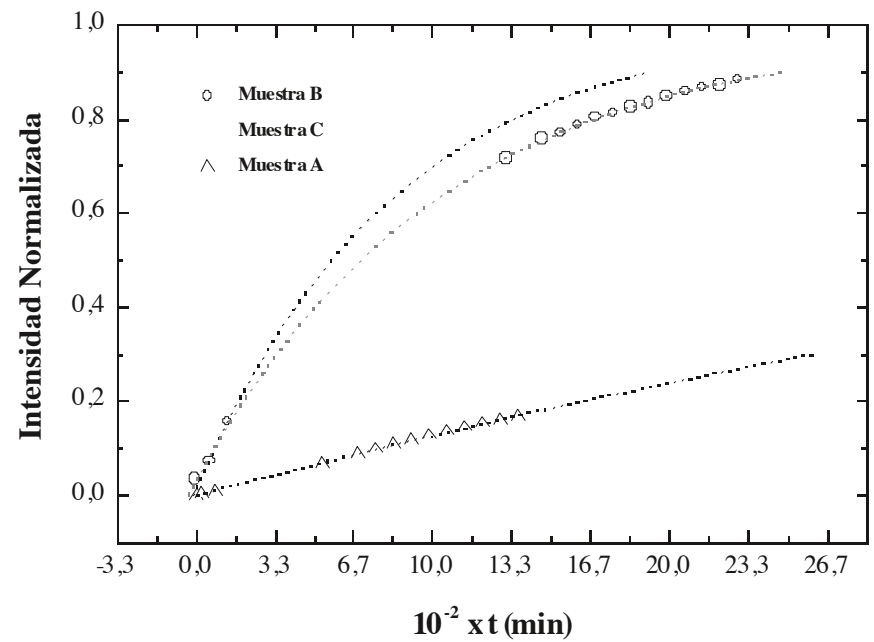

Figura 5.- Variación de la relación de intensidades $\left(\mathrm{I}_{\mathrm{PSA}} / \mathrm{I}_{\text {ovaleno }}\right)$ normalizada, para las tres muestras en estudio, en función del tiempo de permanencia de las fibras en disolución ( $\mathrm{T}=293 \mathrm{~K}$ ).
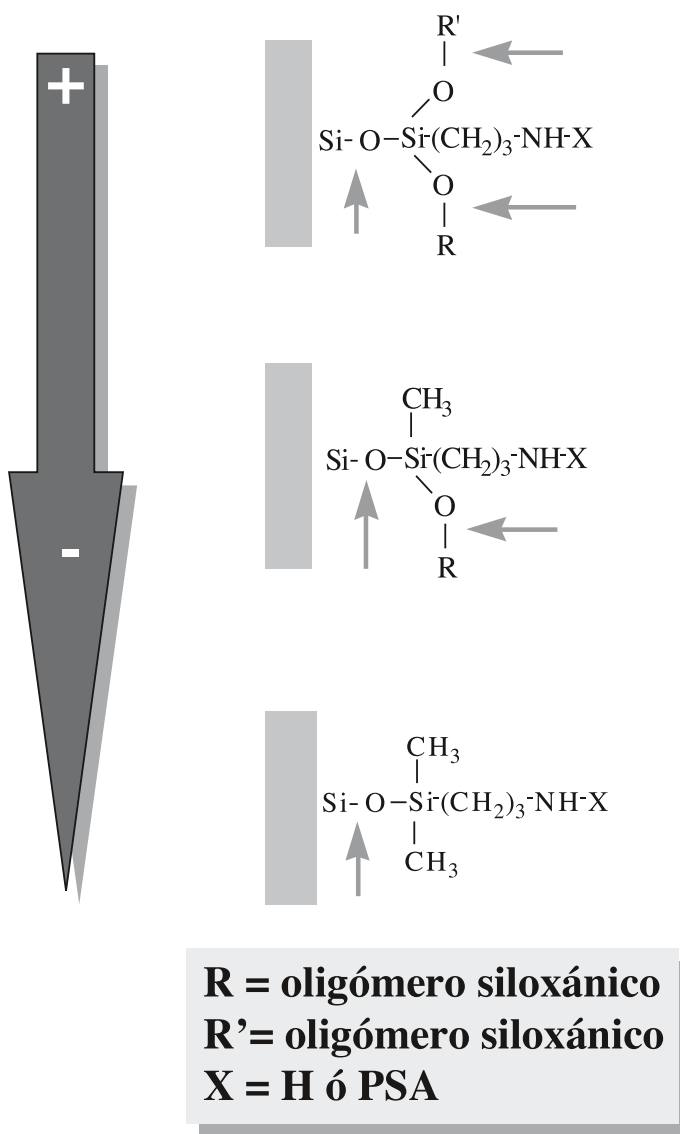

Figura 6.- Esquema representativo de la relación existente entre tipo de enlace siloxánico y velocidad de degradación hidrolítica. 
10. L. Salmon, I. Thominette, M.F. Pays, J. Verdu, Hydrolitic Degradation of Model Networks Simulating the Interfacial Layers in Silane-Coupled Epoxy/ Glass Composites, Composites Science and Technology, 57, 1119-1127, (1997).

11. H. Ishida; J.L. Koenig; The Reinforcement Mechanism of Fiber-Glass Reinforced Plastics Under Wet Conditions: A Review, Polymer Engineering and Science, 18, 2, 128-145, (1978)

12. H. Hamada, N. Ikuta, N. Nishida, Z. Maekawa, Effect of Interfacial silane network structure on interfacial strength in glass fiber composites, Composites, 25, 7, 512-515, (1994).

13. J. González-Benito, Modificación Superficial de Fibras de Vidrio. Microestructura, Propiedades y Degradación, Tesis Doctoral, Dpto. Química Física I, U.C.M, (1999).

14. R.K. Iler, The Chemistry of Silica, Pg. 33, John Wiley \& Sons, New York, (1979). 